\title{
The prognosis of hospital-referred transient ischaemic attacks
}

\author{
Graeme J Hankey, James M Slattery, Charles P Warlow
}

\begin{abstract}
A cohort of 469 hospital-referred patients with transient ischaemic attacks (TIA) of the brain $(66 \%)$ or eye $(34 \%)$ due to presumed atheromatous thromboembolism, lipohyalinosis or cardiogenic embolism, without prior stroke, was assembled between 1976-86. Follow up was prospective and complete until the patients death or the end of 1986 . During a mean period of follow up of $4 \cdot 1$ years there were 82 deaths ( 58 vascular, 24 non-vascular), 63 first-ever strokes and 58 patients with coronary events. A coronary event accounted for $51 \%$ of deaths whilst stroke was the cause in $12 \%$. The average risk of death over the first five years after TIA was $4.5 \%$ per year. The risk of stroke was $6.6 \%$ in the first year and $3.4 \%$ per year on average over the first five years. Stroke occurred in the same vascular territory as the initial TIA in about two-thirds of cases, and was of lacunar type in one fifth of these strokes. The average risk of a coronary event over the first five years after TIA was $3 \cdot 1 \%$ per year, similar to that of stroke. However, the risk of a coronary event, and also death, was fairly constant each year after a TIA, in contrast to the risk of stroke which was highest in the first year. The average risk of stroke, myocardial infarction or vascular death over the first five years after TIA was $6.5 \%$ per year and the average risk of stroke, myocardial infarction or death from any cause was $7 \cdot 5 \%$ per year. The prognosis of this cohort of hospitalreferred TIA patients was better than that of TIA patients in the same community who presented to the Oxfordshire Community Stroke Project (OCSP), and reflected the impact of referral bias. The hospital-referred patients were younger, assessed at a later date after their last TIA, and comprised a greater proportion of patients who had had a TIA of the eye (amaurosis fugax), which had a better prognosis than TIA of the brain. Knowledge of the prognosis of different populations of TIA patients not only enhances understanding and interpretation of previous studies but is also required for optimal patient management and the planning of treatment trials.
\end{abstract}

The natural history of transient ischaemic attacks of the brain and eye (TIA), without medical intervention, is unknown. This is because most patients with TIA receive some form of treatment (for example, antihypertensive, antiplatelet, anticoagulant, carotid endarterectomy) which influences outcome, whilst other patients have never reported, nor perhaps even noticed, such transient symptoms. The prognosis of TIA, describing the outcome of TIA patients who have come under medical care and who have been treated in a variety of ways that may have affected the subsequent course of events, has been the subject of several studies with differing methods and results. ${ }^{1-19}$ The sources of variation include: a) the population (patient sample) examined: this may be community 58101119 or hospital-based, ${ }^{1-46912-18}$ with differences in referral patterns, patient selection, age structure, the presence and level of vascular diseases and risk factors, and the presence $e^{1415}$ or absence $e^{5-810-12}$ of a history of previous stroke; b) the size of the sample: many studies are limited by small numbers ${ }^{1-311}$ and consequently estimates of prognosis have wide confidence intervals; $c$ ) the definition of TIA: some studies have only included patients with TIA symptoms of one hour ${ }^{4}$ or four hours ${ }^{3}$ duration while other studies have included patients with symptoms lasting up to 72 hours and others have excluded patients with symptoms lasting less than 15 minutes; ${ }^{6}$ some studies have examined only patients with their first TIA $^{581013}$ or an "incident" TIA, ${ }^{19}$ whereas others have studied patients who presented with a TIA, whether or not it is their first, and some studies have even included patients with merely non-focal symptoms; ${ }^{1}$ d) methodology: prospective cohort study ${ }^{267911141517-19}$ or retrospective case series study; ; $^{13810121316} \mathrm{e}$ ) treatment: various medical and/or surgical treatments; $f$ ) definition and assessment of outcome events, such as stroke; g) length and adequacy of follow up; and h) analysis: many studies have expressed the outcome of TIA patients in very simple terms: the number of deaths or strokes occurring during the average period of follow up divided by the original total number of patients. This method fails to consider the timing of the event, such as stroke, during follow up (for example, it does not distinguish between a stroke occurring within five days of a TIA from a stroke occurring five years later) nor the outcome of patients who were "lost" to follow up or who died, for example, from other perhaps more frequent causes (such as cardiac deaths), and who were therefore no longer at risk of stroke. The actuarial life table ${ }^{20}$ and Kaplan-Meier product limit ${ }^{21}$ techniques both allow for censored data and generally produce very similar survival curve estimates. Given the differences 
in duration and adequacy of follow up amongst the studies, it is almost impossible to compare the results of studies which did not use actuarial methods of analysis.

Furthermore, although TIAs (and some of the outcome events, such as stroke) have usually been studied as a homogeneous group, they are heterogeneous in many respects, such as pathogenesis (for example, TIA due to hyperviscosity or arteritis, as opposed to thromboembolism). It is likely that TIAs of different pathogenesis also have a different prognosis.

Not only is it difficult to compare the results of many of the previous studies of TIA prognosis (particularly those with few cases, inadequate follow up and without actuarial analysis of the survival data) but reports of TIA prognosis from different periods, even within the same medical institution, may not be comparable. ${ }^{22}$

The only published prospective and community-based study of the prognosis of TIA which had enough patients to provide a reasonable estimate of prognosis was carried out in Oxfordshire, United Kingdom between $1981-86 .{ }^{19}$ During a similar period (1976-86), and in the same community, one of us (CPW) prospectively evaluated and followed up a large hospital-referred series of patients with TIA. Standardised diagnostic and outcome criteria were applied prospectively in both populations and all outcome events were analysed using actuarial methods. It is therefore possible to compare and contrast the prognosis of TIA patients who are seen by a general practitioner in the comunity with the prognosis of TIA patients who are seen in a hospital by a consultant neurologist, and to study the influence of referral bias on the results.

This series is a descriptive account of prognosis. Later, we shall be reporting on which baseline variables, such as hypertension, angiographic evidence of carotid stenoses, affect prognosis. We shall also be reporting a predictive model of prognosis that has been derived from the important prognostic factors in these hospital-referred patients which may apply to other TIA populations. Our results can be used for advising patients treated similarly to ours, on their risk of various vascular events and in the planning of clinical trials.

\section{Subjects and methods}

Between 1977-86 we assembled a cohort of 481 consecutive patients presenting to one of us (CPW) with TIA but no history of previous stroke: 319 patients $(66 \%)$ presented with transient cerebral ischaemic attacks (TCIA) and 162 with transient ischaemic attacks of the eye (amaurosis fugax, [AFx]). Patients were referred at an early and fairly uniform point in the course of their disease, quite soon after the onset of their symptoms and before any stroke had occurred. The referral source was the patient's general practitioner in $58 \%$ of cases,

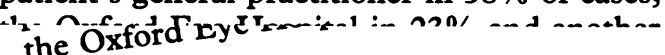

hospital consultant in $19 \%$. Standard diagnostic and outcome criteria (appendix) were applied and patients who were considered to have a diagnosis of migraine, epilepsy or transient global amnesia were excluded. Complete follow up was achieved by way of either regular clinical review at four to 12 monthly intervals until the patient died or the end of 1986 ( $n=467)$, or by writing to the patient and/or the general practitioner $(n=14)$. All patients who had a vascular event of any consequence were evaluated by CPW and all available records, including necropsy reports, were reviewed. A second observer (GJH) reviewed the records at the end of the study.

Cerebral angiography was carried out if a patient was a potential candidate for carotid endarterectomy on clinical grounds (that is, carotid TIA and medically fit for surgery) and had consented to surgery if the angiogram showed a potentially operable lesion of the internal carotid artery (ICA). The decision to proceed to cerebral angiography was not influenced by the presence or absence of a carotid bruit. Non-invasive carotid ultrasound studies were not available to screen and select patients for angiography.

\section{Statistical analysis}

Survival analysis of the TIA population was confined to 469 of the 481 patients ( $98 \%$ ) in whom the cause of the presenting TIA was considered to be atheromatous arterial thrombosis, causing thromboembolism or haemodynamic compromise, lipohyalinosis or cardiogenic embolism. Eight patients with arteritis, three patients with focal cerebral ischaemia caused by cardiac arrhythmia and one patient with carnio-cervical trauma were excluded from the survival analysis. Neurological consultation with CPW was taken as day 0 of follow up. As this is a study of prognosis and not untreated natural history, we have included all patients, whether treated (with aspirin, carotid surgery, etc) or not. The Kaplan-Meier technique of survival analysis was used. ${ }^{21}$ The average annual risk $(\mathrm{z} \%)$ of each outcome event was calculated as follows: $\mathrm{z}=100\left[1-(1-\mathrm{y} / 100)^{1 / 5}\right) \%$, where $\mathrm{y}=$ the percentage risk of the outcome event during the first five years of follow up. A $95 \%$ confidence interval (CI) was calculated for the proportion " $y$ " by multiplying the standard error of " $y$ " by 1.96 and adding and subtracting the product from " $y$ ". ${ }^{23}$ The $95 \%$ confidence interval for the average annual rate of each outcome event was obtained by substituting " $y$ " in the above formula with the upper and lower $95 \%$ confidence limit of " $y$ " successively. As many previous studies of TIA prognosis were characterised by incomplete follow up and analysis of results using non-actuarial methods, it was not possible to derive an accurate risk of survival in those studies because the patients who were lost to follow up could not be accounted for and were not censored. The studies in which the results were analysed using actuarial techniques have produced accurate survival rates (at five years,

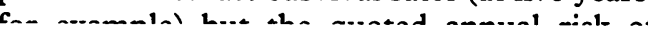


death (and other events) was usually the arithmetic average annual risk, which was calculated by simply dividing the risk over the follow up period by the length of follow up in years. This linear approximation of the average annual risk gives a slight underestimate of the average annual risk, particularly when the risk is large. The average annual risk of various outcome events over the first five years after TIA has been recalculated, using the above formula, for the large prospective studies that provided actuarial estimates of outcome (table 4). ${ }^{141718}$

The odds ratios (OR) and $95 \%$ confidence intervals for odds ratios were calculated using standard methods. ${ }^{23}$

\section{Results}

Baseline Characteristics

As in most hospital series of patients with TIA, these 469 patients (TCIA: $n=311$, AFx: $\mathrm{n}=158$ ) were elderly, predominantly male, and characterised by a high prevalence of vascular disease and risk factors (table 1 ). The median time interval between the last TIA and evaluation by the neurologist was 10 days (interquartile range 25 days 4-29 days).

At presentation, $144(31 \%)$ patients were being treated with drugs for hypertension, eight were taking aspirin, four were on dipyridamole and two were on oral anticogulant medication.

Cranial CT was performed on 302 patients (64\%). CT was normal in 227 patients $(75 \%)$ and showed evidence of widespread cortical loss $(\mathbf{n}=33)$ and/or a focal area of hypodensity $(n=52)$ in the other 75 patients $(25 \%, 95 \%$ CI: 20 to $30 \%$ ). The focal area of low density was in an area appropriate to the patient's symptoms in 26 patients ( $9 \%, 95 \%$ CI: 6 to $12 \%$ ), 23 of whom presented with ipsilateral TCIA and three with ipsilateral AFx. In 26 patients CT showed a focal region of low density in an area unrelated to the patient's symptoms. Cerebral angiography was performed in 229 patients ( $49 \%$ ), and seven postangiographic complications occurred in six (local 1, systemic 2, transient neurological 2, minor stroke 1 , non-disabling major stroke 1 ). The high prevalence of disease in the symptomatic proximal ICA (fig 1) probably represents some selection bias because the asymptomatic carotid system was usually studied angiographically if a potentially operable lesion had been demonstrated by angiography on the symptomatic side.

Table 1 Baseline data in this series compared with TIA patients in a community series ${ }^{32}$

\begin{tabular}{|c|c|c|}
\hline & $\begin{array}{l}\text { Hospital-referred } \\
\text { (Oxford) }\end{array}$ & $\begin{array}{l}\text { Community } 32 \\
\text { (Oxfordshire) }\end{array}$ \\
\hline 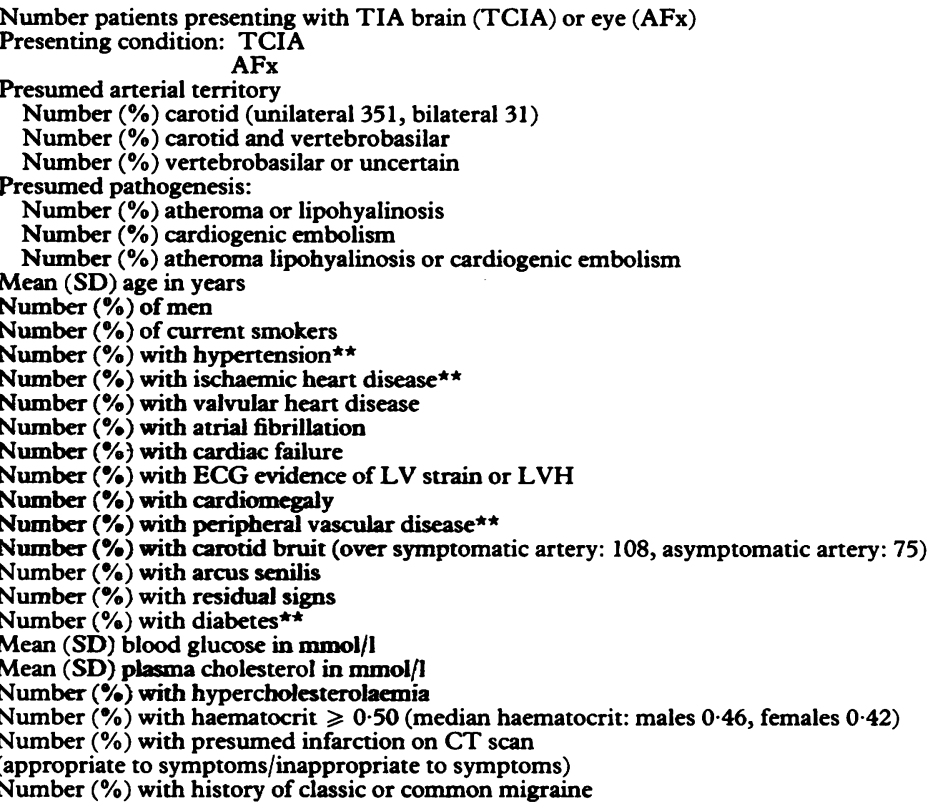 & $\begin{array}{l}469^{\star} \\
311(66) \\
158(34) \\
346(74) \\
36(8) \\
87(19) \\
387(83) \\
34(7) \\
48(10) \\
62 \cdot 1(12) \\
317(68) \\
221(47) \\
198(42) \\
99(21) \\
71(15) \\
18(4) \\
23(5) \\
46(10) \\
76(16) \\
79(17) \\
141(30) \\
221(47) \\
26(6) \\
25(5) \\
5 \cdot 2(1) \\
6 \cdot 7(1.4) \\
201(42) \\
4 \cdot 6(10) \\
52 / 302(17) \\
26 / 26 \\
84(18)\end{array}$ & $\begin{array}{c}21(11) \\
38(21) \\
72(39) \\
0(0) \\
12(6) \\
5 \cdot 6(2) \\
6 \cdot 9(1 \cdot 6) \\
95(52) \\
29(16) \\
32 / 120(27) \\
14 / 18\end{array}$ \\
\hline
\end{tabular}

^Excludes the 12 patients whose TIA was not thought to be due to atheroma, lipohyalinosis (for example, vasculitis)

$\star$ Excludes the 12 patients whose TIA was not thought to be due to atheroma
$\star \star$ Somewhat different definitions of disease/risk factor between the studies

Definitions

Current smoker: current cigarette, pipe or cigar smoker in last 12 months.

Hypertension: diastolic blood pressure $\geqslant 100 \mathrm{mmHg}$ or treated hypertension.

Ischaemic heart disease: history of angina, myocardial infarction or coronary artery bypass surgery.

Valvular heart disease: if the patient had a resting systolic murmur of at least grade $2 / 6$ intensity or any diastolic murmur, or a diagnostic valvular abnormality on echocardiography.

Both auscultatory and echocardiographic evidence was required for the diagnosis of mitral leaflet prolapse.

Atrial fibrillation: confirmed by electrocardiograph (ECG) before or at presentation.

ECG evidence of LV strain: ST segment depression and T wave inversion in leads I, II, AVL, V4-V6.

ECG evidence of LV hypertrophy: the voltage sum of the tallest $R$ wave and the deepest $S$ wave $\geqslant 40 \mathrm{~mm}$ in the praecordial leads, provided the QRS duration $<0.10$ seconds.

Cardiomegaly: the cardiothoracic ratio $>0.5$ on chest radiograph

Peripheral vascular disease: intermittent claudication, ischaemic rest pain or previous peripheral vascular surgery.

Carotid bruit: ${ }^{46}$

Arcus senilis: ring-like opacity at the periphery of the cornea, near the limbus.

Diabetes mellitus: fasting plasma glucose $\geqslant 8.0 \mathrm{mmol} / 1$, random plasma glucose $\geqslant 11.0 \mathrm{mmol} / 1$ or treated diabetes.

Hypercholesterolaemia: fasting plasma cholesterol $\geqslant 7.0 \mathrm{mmol} / \mathrm{l}$ or treated hypercholesterolaemia. 
One hundred and two patients $(22 \%)$ were also included in the Oxfordshire Community Stroke Project (OCSP) series ${ }^{19}$ since they came from the 10 general practitioners collaborating in the OCSP; the remaining patients $(78 \%)$ were referred either before the start of the OCSP or by other practices in and around Oxford.

\section{Follow up}

Complete follow up of every patient was obtained until the end of 1986 or the patient's death if earlier. The mean period of follow up was $4 \cdot 1$ years (range one to 10 years in survivors).

During follow up, hypertension requiring treatment developed in 19 patients $(4 \%)$, angina in $25(5 \%)$, atrial fibrillation in six $(1 \%)$, diabetes in four $(1 \%)$, intermittent claudication in $26(6 \%)$ and cardiac failure in $18(4 \%)$.

\section{Treatment during follow up}

At some stage during follow up, 179 (38\%) patients were treated with drugs for hypertension, 239 patients $(51 \%)$ were treated with aspirin, $54(12 \%)$ were taking placebo in the UK-TIA aspirin trial, ${ }^{24} 11(2 \%)$ were treated with oral anticoagulants, 55 patients $(12 \%)$ had carotid endarterectomy (bilateral in 4), 27 (6\%) were randomised to "no surgery" in the European Carotid Surgery trial, three had extra- to intracranial bypass surgery, six had coronary artery bypass surgery, four had other cardiac surgery and seven had peripheral vascular surgery.

MAJOR EVENTS

The numbers of each of the major outcome events which occurred during follow up are shown in table 2; the average annual risk and actuarial risk at one year and five years of each major event is shown in table 3 and the KaplanMeier survival curves for each major event are seen in figures 2 to 7 .

\section{Stroke}

Seventy six strokes occurred in 63 patients $(13 \%)$. Clinically, there was no doubt about the diagnosis of stroke. ${ }^{25-27}$ Pathologically, 30 strokes were definite infarcts (necropsy: 2 , cranial CT within 28 days: 24 , necropsy and CT: 4 ), 21 were probable infarcts (CT after 28 days), three were definite primary intracerebral haemorrhages (CT within 28 days: 3 ), and 22 were of uncertain type (neither CT, postmortem or Guy's Hospital Diagnostic Score

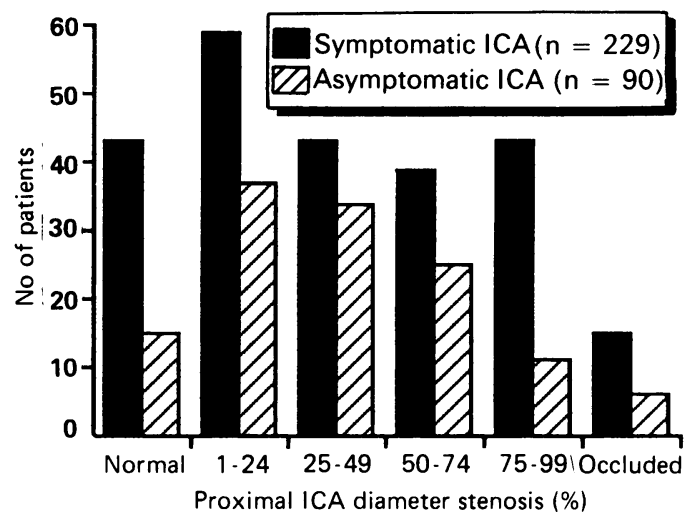

Figure 1 Histogram showing the degree of diameter stenosis of the origin of the internal carotid artery on the symptomatic and asymptomatic sides. The asymptomatic carotid artery was only studied if a potentially operable lesion was demonstrated angiographically on the symptomatic side.

Table 2 Number of outcome events during follow up (mean $4 \cdot 1$ years) of 469 patients with TIA

\begin{tabular}{|c|c|c|c|c|c|}
\hline Outcome events & $\begin{array}{l}\text { TCIA }(n=311) \\
\text { Number }\end{array}$ & $\begin{array}{l}\text { AFx }(n=158) \\
\text { Number }\end{array}$ & $\begin{array}{l}\text { TCIA or } \\
\text { Number }\end{array}$ & $\underset{\%}{\mathrm{AFx}}(\mathrm{n}$ & $\begin{array}{l}=469) \\
95 \% C I s\end{array}$ \\
\hline Death & 57 & 25 & 82 & 17 & 14 to 21 \\
\hline Vascular death & 44 & 14 & 58 & 12 & 9 to 15 \\
\hline Stroke & 10 & 0 & 10 & & \\
\hline Coronary Event & 30 & 12 & 42 & & \\
\hline Other vascular ${ }^{\star}$ & 4 & 2 & 6 & & \\
\hline Non-vascular death & 11 & 10 & 21 & 4 & 3 to 6 \\
\hline Unknown & 2 & 1 & 3 & 1 & 0 to 3 \\
\hline Stroke $\star \star$ & 49 & 14 & 63 & 13 & 10 to 17 \\
\hline Minor & 13 & 3 & 16 & & \\
\hline Major non-fatal & 38 & 12 & 50 & & \\
\hline Non-disabling & 28 & 9 & 37 & & \\
\hline Disabling & 10 & 3 & 13 & & \\
\hline Major fatal & 9 & 1 & 10 & & \\
\hline Retinal infarction & 4 & 1 & 5 & 1 & 0 to 3 \\
\hline Coronary event & 41 & 17 & 58 & 12 & 9 to 15 \\
\hline Disabling major stroke/vascular death & 54 & 17 & 71 & 15 & 12 to 18 \\
\hline Stroke/MI/vascular death & 88 & 30 & 118 & 25 & 21 to 29 \\
\hline Stroke $/ \mathrm{MI} /$ death & 98 & 34 & 132 & 28 & 24 to 32 \\
\hline
\end{tabular}

$\star$ Deaths due to rheumatic heart disease, cardiomyopathy, ruptured aortic aneurysm, etc.

$\star \star 63$ patients suffered a total of 76 strokes

TCIA: transient cerebral ischaemic attack

AFx: amaurosis fugax

TIA: transient ischaemic attack of the brain or eye

MI: myocardial infarction

Table 3 Summary of risks of the different measures of outcome for TIA patients

\begin{tabular}{|c|c|c|c|c|c|}
\hline Outcome event & $\begin{array}{l}\text { Actuar } \\
1 \text { year }\end{array}$ & $\begin{array}{l}\text { isk at } \\
5 \text { years }\end{array}$ & $95 \% C I$ & $\begin{array}{l}\text { Average annual risk } \\
\text { (over the first } 5 \text { years) }\end{array}$ & $95 \% C I$ \\
\hline $\begin{array}{l}\text { Death } \\
\text { Stroke } \\
\text { Coronary event } \\
\text { Disabling major stroke or vascular death } \\
\text { Stroke, MI or vascular death } \\
\text { Stroke, MI or death }\end{array}$ & $\begin{array}{l}3 \cdot 4 \% \\
6 \cdot 6 \% \\
2 \cdot 5 \% \\
4 \cdot 5 \% \\
9 \cdot 0 \% \\
9 \cdot 5 \%\end{array}$ & $\begin{array}{l}20 \cdot 5 \% \\
16 \cdot 0 \% \\
14 \cdot 6 \% \\
18 \cdot 3 \% \\
28 \cdot 6 \% \\
32 \cdot 1 \%\end{array}$ & $\begin{array}{l}16 \text { to } 25 \% \\
12 \text { to } 20 \% \\
11 \text { to } 18 \% \\
14 \text { to } 23 \% \\
24 \text { to } 33 \% \\
27 \text { to } 37 \%\end{array}$ & $\begin{array}{l}4 \cdot 5 \% \\
3 \cdot 4 \% \\
3 \cdot 1 \% \\
4 \cdot 0 \% \\
6 \cdot 5 \% \\
7 \cdot 5 \%\end{array}$ & $\begin{array}{l}3.4 \text { to } 5.6 \% \\
2.5 \text { to } 4.6 \% \\
2.2 \text { to } 4.0 \% \\
3.0 \text { to } 5.0 \% \\
5.3 \text { to } 7.8 \% \\
6.2 \text { to } 8.8 \%\end{array}$ \\
\hline
\end{tabular}

MI: myocardial infarction 
Figure 2 Kaplan-Meier survival curve showing the percentage survival during the first eight years after a TIA. The vertical error bars indicate the $95 \%$ confidence interval of the estimate at each point in time after the TIA.

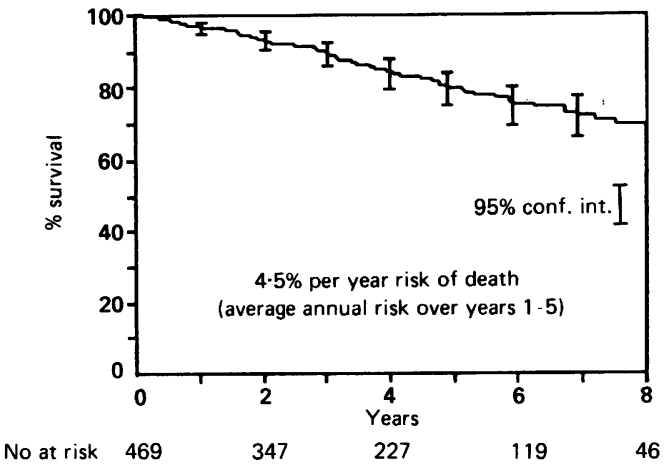

Figure 3 Kaplan-Meier survival curve showing the percentage survival free of stroke (censoring those dying from causes other than stroke) during the first eight years after a TIA. The vertical error bars indicate the $95 \%$ confidence interval of the estimate at each point in time after the TIA.
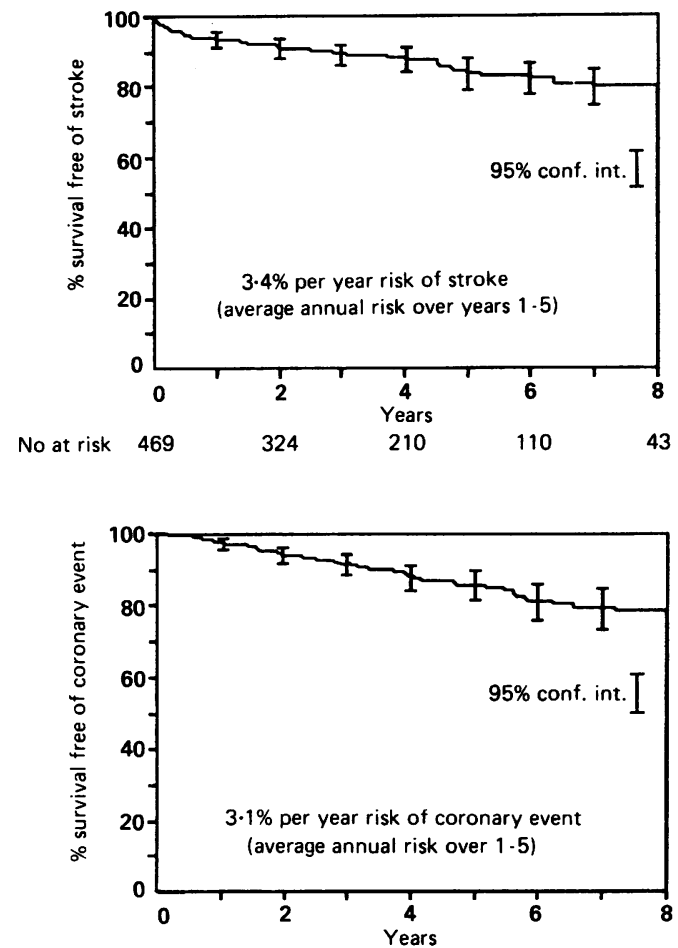

Figure 4 Kaplan-Meier survival curve showing the percentage survival free of a coronary event (censoring those dying from causes other than a coronary event) during the first eight years after a $T I A$. The vertical error bars indicate the $95 \%$ confidence interval of the estimate.
Figure 5 Kaplan-Meier survival curve showing the percentage survival free of a disabling stroke or vascular death (censoring those dying from causes other than a vascular death) during the first eight years after a TIA. The vertical error bars indicate the $95 \%$ confidence interval of the estimate.

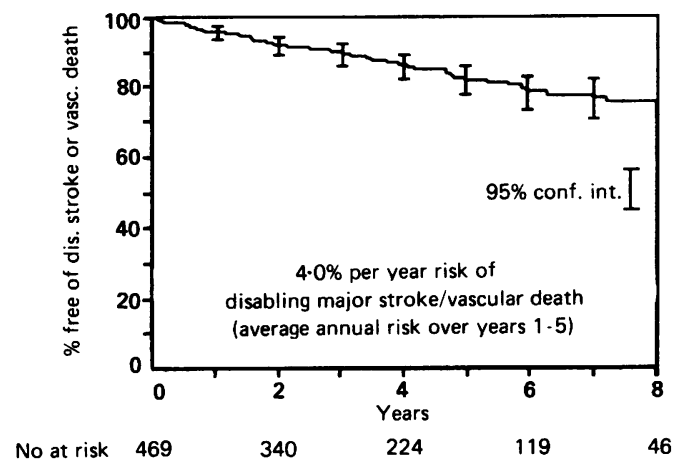

Figure 6 Kaplan-Meier survival curve showing the percentage survival free of a stroke, myocardial infarction or vascular death (censoring those dying from causes other than a stroke, myocardial infarction or vascular death) during the first eight years after a TIA.

The vertical error bars indicate the $95 \%$

confidence interval of the estimate.

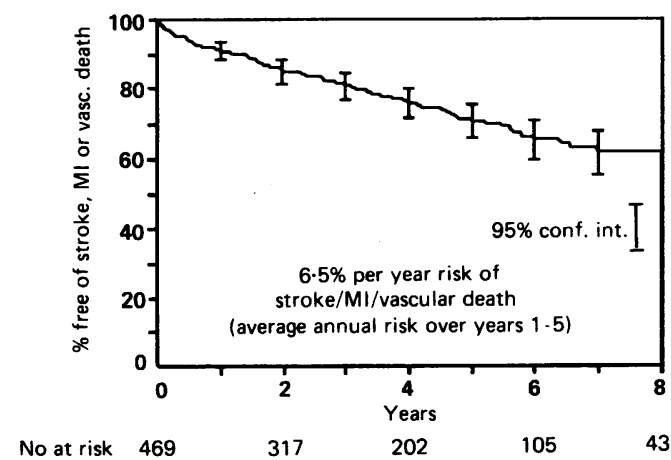

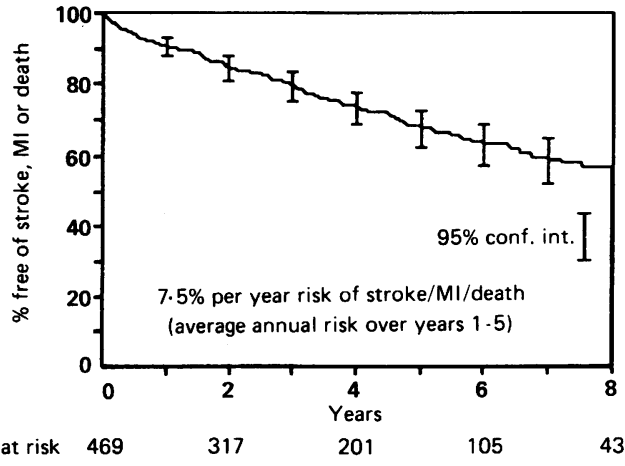

Figure 7 Kaplan-Meier survival curve showing the percentage survival free of a stroke, myocardial infarction or death during the first eight years after a TIA. The vertical error bars indicate the $95 \%$ confidence interval of the estimate.

$<4$ or $>24) .{ }^{27}{ }^{28}$ Ten strokes were fatal, 50 were non-fatal major (of which 13 were disabling ${ }^{29}$ and 16 were minor. Stroke occurred in the same vascular territory as the presenting TIA in 40 of the 63 patients $(63 \%), 30$ of whom had presented initially with TCIA and 10 of whom had presented with AFx. Stroke in the same vascular territory was of lacunar type clinically ${ }^{30}$ in eight of these 40 patients $(20 \%)$. Of the 10 first-ever strokes occurring within 30 days of presentation, six occurred either within 24 hours of cerebral angiography $(n=2)$ or within 24 hours of carotid endarterectomy $(n=4)$. These strokes were included in the survival analysis.

\section{Coronary Event}

Seventy three coronary events occurred in 58 patients $(12 \%)$, of which 42 were fatal (table 2 ). Non-fatal myocardial infarction occurred on 31 occasions in 26 patients, fatal myocardial infarction in 15, sudden death in 18 and fatal cardiac failure secondary to ischaemic heart disease in nine patients. In addition, three patients died from a non-ischaemic cardiomyopathy and three from a ruptured aortic aneurysm.

\section{MINOR EVENTS}

After presention, TCIA occurred/recurred in $102(22 \%)$ patients (median 3 TIAs, range $1-$ 50 ) and $69(15 \%)$ suffered $A F x$ (median 3 , range 1-15). Subsequent TCIA and/or AFx occurred in 165 patients $(35 \%)$. Retinal infarction developed in five patients $(1 \%)$, four of whom had presented with TCIA (ipsilateral carotid territory in three) and one with ipsilateral AFx.

\section{Discussion}

Study sample

We describe the prospective longitudinal study of a cohort of patients with no previous history of stroke who experienced a TIA due to presumed atheromatous thromboembolism, lipohyalinosis or cardiogenic embolism and were referred to a single neurologist (CPW) at an early and relatively uniform point (inception) in the course of their disease. Since this is a study of the prognosis and not natural history of TIA, all patients were included in the 
analysis, irrespective of medical or surgical therapy. Transient ischaemic attacks of the eye (AFx) were included with those to the brain (TCIA) because it was considered that the underlying pathogenesis was very similar and that they should probably be regarded as a single entity in pathophysiological and also possibly prognostic terms. Patients were excluded from entry into the study if they had already had a stroke before neurological assessment, irrespective of whether it occurred before the TIA or between the TIA and subsequent neurological consultation. The inclusion of such patients would have biased the group towards a worse prognosis than was the case; the reason being that patients with TIA are more likely to present to medical attention if the TIA is followed by a stroke. The same criteria were applied in the study of prognosis of patients with TIA in the Oxfordshire Community Stroke Project (OCSP). ${ }^{19}$

\section{Referral bias}

This sample of hospital-referred TIA patients differs from TIA patients in the general population due to selection of patients at each stage of the referral process and consequent referral bias. Patient referral was determined in part by our particular interest in patients with TIA. Although a community-based study should reflect the characteristics of the general population, the OCSP data are also incomplete, but less so, because not all patients with TIA seek medical attention. ${ }^{31}$ It is therefore impossible to know the true incidence and prognosis of TIA in the community but the OCSP study perhaps provides the best current estimate.

Although 102 TIA patients in the OCSP study were referred and therefore also included in this hospital-referred cohort, such overlap had not negated the significant differences between the two TIA populations in terms of age, sex, presenting complaint, prevalence of cigarette smokers and atrial fibrillation, level of haematocrit and time to assessment (table 1 and fig 8). The referred patients were younger and characterised by a definite excess of men com-

Figure 8 Diagram
showing the odds ratio
(OR), with its $95 \%$
confidence interval (CI),
of hospital-referred
patients with a transient
ischaemic attack (TIA)
possessing a vascular
disease or risk factor
compared with patients
with a TIA in the
community. ${ }^{31}$ If the OR
is $1 \cdot 0$, then the prevalence
of the risk factor is
approximately equal in the
two groups.
MI: myocardial
infarction.

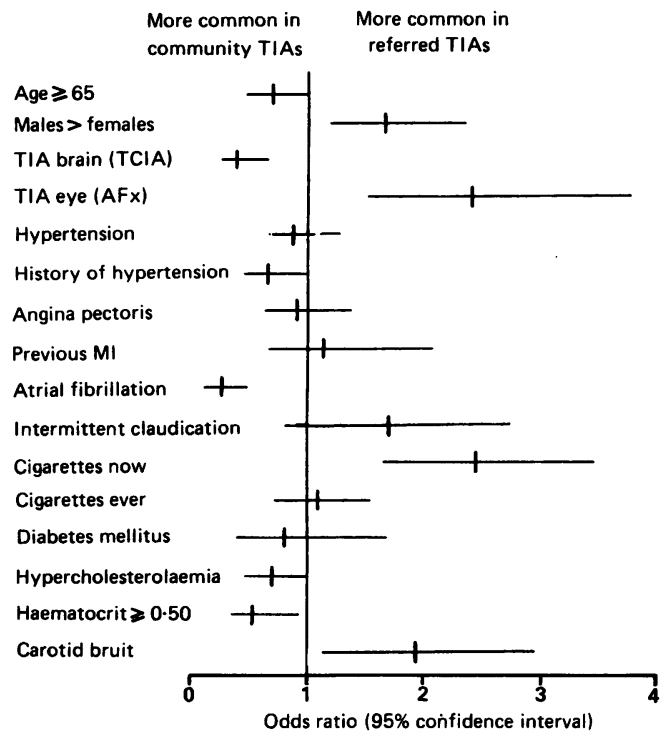

pared with the community study. This significant difference in sex ratio is probably due to the lack of elderly patients (who are less commonly referred to hospital) and therefore women in our series, since the risk of having a TIA or stroke is greater in middle aged men than in women of the same age..$^{25} 263132 \mathrm{~A}$ higher proportion of patients in the hospital-referred series presented with $A F x$, which reflects the referral patterns of the Oxford Eye Hospital. The younger, hospital-referred patients had a greater prevalence of current cigarette smokers and the older community sample had a greater prevalence of atrial fibrillation and higher haematocrit levels. Other studies have shown that the prevalence of cigarette smokers declines in older age groups while the prevalence of atrial fibrillation increases with age. ${ }^{33-36}$ The time between the onset of the TIA and assessment by the general practitioner and subsequently the neurologist, was greater for our hospital-referred patients (10 days versus three days, medians), during which time a serious vascular event may have occurred. No great difference in treatment existed between the two populations; in the referred and community samples the respective use of aspirin was $51 \%$ and $57 \%$, antihypertensive therapy: $38 \%$ and $44 \%$, and carotid endarterectomy: $12 \%$ and $3 \%$.

\section{Diagnosis}

The diagnosis of TIA is clinical and requires the skill of an experienced physician; it cannot be confirmed or rejected by any objective test or procedure and is therefore susceptible to both intraobserver and interobserver variation. Even among trained neurologists, the interobserver agreement for the diagnosis of a cerebral TIA is not very good (kappa $=0.65$ ); and it is even worse for the arterial territory (kappe $=0.31) .{ }^{37} 38$ Our sample of patients was diagnosed by a single observer, experienced in the assessment of patients with TIA, using standardised diagnostic criteria, and the diagnoses were reviewed by a second observer.

It has been suggested that patients with TIA who have an appropriately sited area of low density on CT should be reclassified as cerebral infarction with transient signs (CITS) rather than a TIA. ${ }^{3940}$ We disagree, and have not reclassified our TIA patients, with CT evidence of appropriately-sited low density areas, as having a stroke, because: 1) the low density lesion may not be due to infarction occurring at the time of the TIA (it may be an old haemorrhage, for example); 2) the detection of ischaemic lesions on CT depends on the timing and resolution of the scan; 3 ) a CT scan would become essential for the diagnosis of TIA; 4) the diagnosis of TIA would then become difficult to standardise because technology varies between centres; some have no imaging, some have CT and others have MRI, which is far more sensitive, and 5) a new diagnostic category would have to be made for patients with a clinically definite stroke who have a normal CT. Also, in a recent prospective, community-based study of 120 patients with TIA, no significant differences were present in 
either the clinical features, duration of attacks or prognosis of the patients with and without low density lesions on $\mathrm{CT}{ }^{41}$

\section{Prognosis (natural history)}

Almost all patients who present for medical attention following a TIA receive some form of medical treatment, so it is not possible to study the natural history of TIA. This and other studies illustrate the prognosis of TIA patients who have received various "modern" treatments.

The most methodologically sound studies of the prognosis of TIA are those in which the data have been acquired prospectively. ${ }^{2467911141517-19}$ Those with the least bias are community-based:578101119 hospital-based studies fail to consider patients who were not referred to hospital and reflect referral bias. ${ }^{1-46912-18}$ Retrospective reviews of case notes are hampered by a difficulty in applying strict diagnostic criteria uniformly and by inadequate follow up. ${ }^{135810121316}$ In table 4, our results are compared with the only large prospective community-based prognostic study of TIA $^{19}$ and three other large prospective hospital-based series in which the survival data have been analysed using actuarial methods. ${ }^{14} 1718$ Although more than a quarter $(28 \%)$ of the patients studied by Sorensen et $a l^{18}$ had presented with a "reversible ischaemic attack" it is unlikely that this introduced significant heterogeneity from a prognostic viewpoint as the prognosis (and also prevalence of vascular disease and risk factors) in patients with minor ischaemic stroke is probably very similar to TIA. ${ }^{32} 42$

\section{Mortality}

The average annual absolute mortality rates in all of the hospital-referred series were about 4 $5 \%$ per year. ${ }^{141718}$ This contrasts with the higher rate $(7 \%)$ in the community, ${ }^{19}$ which may be due to differences in the age of the patients. Between one and two thirds of all deaths were due to coronary events and about $20 \%$ of deaths were due to stroke. In the community TIA patients, the relative risk of death during follow up (mean 3.7 years) was slightly greater $(1 \cdot 4: 1)$ than the expected risk for age and sex-matched people without TIA. ${ }^{19}$ Retrospective community-based studies in Rochester, Minnesota ${ }^{810}$ indicated that the relative risk of death was 9.6 at one month, 2.4 at one year, and about 1.5 after two years. ${ }^{43}$ The high risk at one month is quite striking but the nature of those early deaths are not known, nor the $95 \%$ confidence intervals of the estimate.

\section{Stroke}

The average annual risk of stroke during the first five years after a TIA was about $3-5 \%$ for

Table 4 Prospective studies of prognosis of TIA, using actuarial analysis

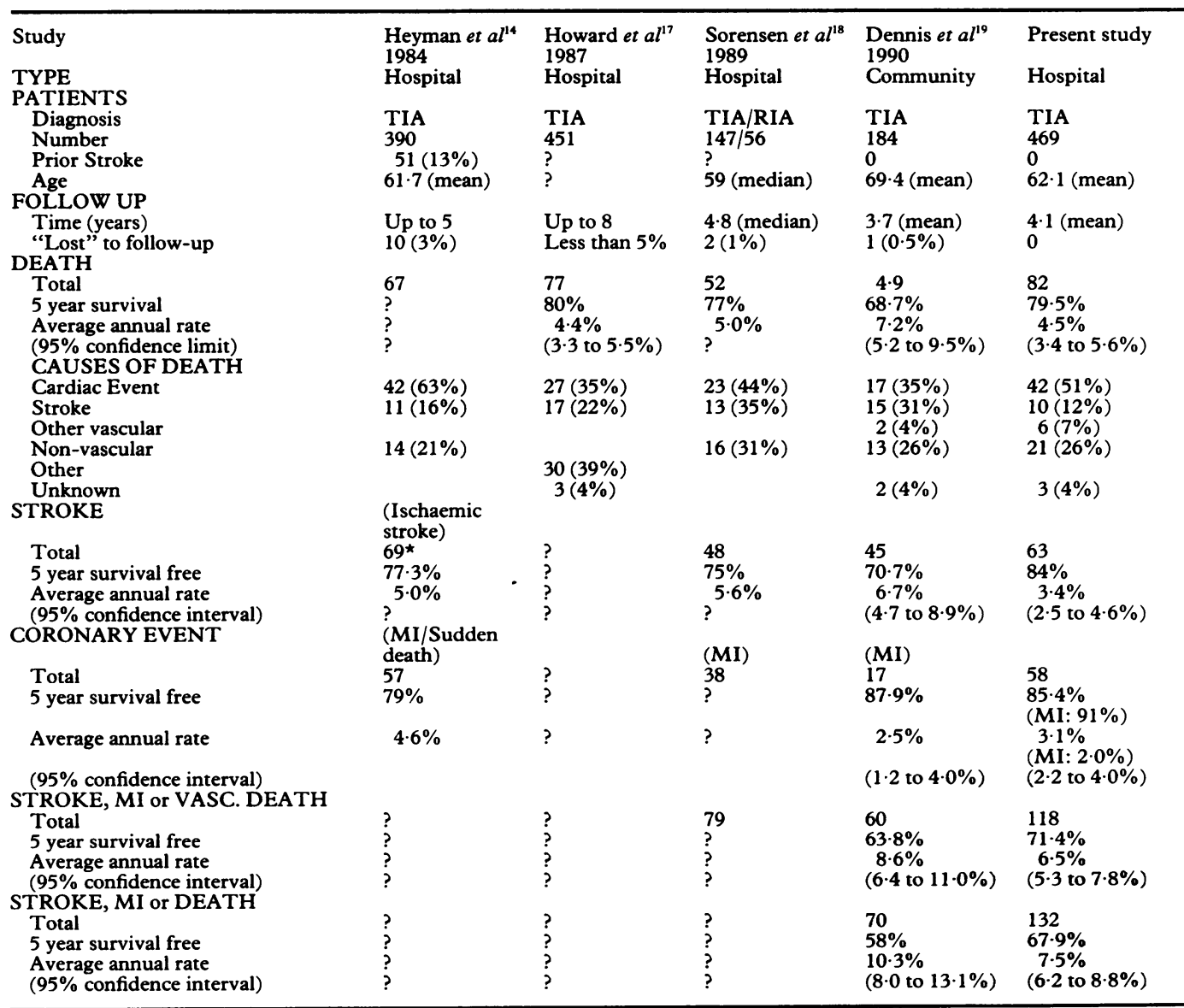

^ 16 strokes were post-angiography or endarterectomy complications

(there were 12 minor strokes and four retinal infarcts which were not included in the analysis)

RIA: reversible ischaemic attack.

MI: myocardial infarction.

Note: the average annual rate in the other studies was recalculated (for formula, see methods section). 
hospital-referred patients and about $7 \%$ in the OCSP. ${ }^{14}{ }^{17-19}$ In our study, the risk of stroke was highest within the first month (about $2 \%$ ) and first year (about 7\%) after TIA and fell thereafter to average about $2-3 \%$ per year for the next five years. The rate of stroke in the first month depends on several factors such as the time of entry into the study and the postangiographic and post-surgical complication rates. In the retrospective studies from Rochester, Minnesota, ${ }^{81043}$ day 0 of follow up was the date of the first-ever TIA, irrespective of whether the patient had come to medical attention or not at that time, and the stroke rate at 30 days was $8 \%$ (95\% CI: $4 \cdot 2$ to $11 \cdot 8 \%)$. In the OCSP, ${ }^{19}$ day of follow up was taken as the day of notification to the study by the general practitioner (median three days after TIA) and the stroke rate at 30 days was $4.4 \%(95 \% \mathrm{CI}$ : 1.5 to $7.3 \%$ ). In our study, day 0 was the day of neurological consultation (median 10 days after TIA), and the 30 day stroke rate was $2 \cdot 1 \%$ (95\% CI: 0.8 to $3.5 \%)$. These results illustrate the approximate risk of stroke for TIA patients from the point of view of awareness to the patient, to the general practitioner and to the neurologist, respectively. In the study by Heyman et al,,$^{14}$ one third (23/69) of all strokes occurred during the initial hospital admission and $70 \%$ of these $(16 / 23)$ were post-angiography or post-endarterectomy complications; quite similar to our iatrogenic stroke rate of $60 \%$ at one month $(6 / 10)$. Although a significant proportion of the early strokes after TIA are complications of angiography or surgery, some other patients with TIA and subsequent early stroke probably have unstable and "active" atherosclerotic plaque in the symptomatic artery. The immediate institution of secondary prevention measures that are targeted to the cause may be critical in these patients.

\section{Type and severity of stroke}

In this study and the OCSP, ${ }^{19}$ only about two thirds of (first-ever) strokes occurred in the same vascular territory as that of the presenting TIA and, of these, about $20 \%$ were of lacunar type, due to presumed small vessel disease. About two thirds of strokes were non-disabling and one-third were disabling or fatal. Therefore, even if carotid endartetectomy is $100 \%$ effective (in preventing non-lacunar infarction in the distribution of the symptomatic carotid artery), it is only likely to prevent about a half of all strokes after TIA, most of which are non-disabling.

\section{Coronary Event}

The average annual risk of a coronary event during the first five years after a TIA was about the same as that of stroke in the hospitalreferred studies ${ }^{141718}$ (particularly if the post angiographic and endarterectomy strokes were excluded). The lack of any excess early risk of a coronary event (compared with stroke) probably reflects the lack of iatrogenic coronary events. Also, we assume that in some patients at least, an "active' atherosclerotic plaque in the cerebral circulation has caused the TIA and is likely to cause a stroke soon after.

The occurrence of TIA is as much a predictor of a cardiovascular event as it is of a cerebrovascular event and therapeutic endeavours need to be directed with equal energy and resources into the prevention of ischaemic cardiac events as ischaemic cerebral and ocular events.

\section{Other serious vascular events}

The risk of having a disabling major stroke or vascular death, which is probably what concerns the patient principally, was about $4 \%$ per year over the first five years in our study. The probability of having a serious vascular event, such as a stroke, myocardial infarction or vascular death, which is what is potentially amenable to antithrombotic treatment ${ }^{24}{ }^{44}$ (as well as control of vascular risk factors), was about $6.5 \%$ per year in this hospital-referred series and $8.6 \%$ per year in the community study.

\section{Impact of referral bias on prognosis}

The better prognosis of the hospital-referred population probably reflects: 1 ) their younger age, 2) the greater proportion of AFx (which has a better prognosis than TCIA, ${ }^{19}$ and 3 ) the longer delay until assessment and entry into the study.

\section{Impact of prognostic factors on prognosis}

Most studies, including our own, have considered TIA patients more or less as a single group (although we only studied TIA due to presumed atheromatous arterial disease, lipohyalinosis or cardiac disease). Such analysis ignores the considerable range of prognoses that must be present in any TIA population. The presence of one risk factor, such as age, does not necessarily imply a bad prognosis, only an increased risk. However, as the number of risk factors/poor prognostic factors increases, the prognosis of the patient declines. Differences in the prevalence and level of these adverse prognostic factors and in treatment among different populations of TIA patients may offer some explanation for the variation in reported results of prognosis. It is very difficult to identify which particular factors are responsible for the relatively favourable prognosis of TIA patients in our cohort compared with other hospital-referred studies. Although the mean age of patients was similar among the different studies, information about other prognostic factors and treatments, common to all studies, is generally lacking. Later, we shall be reporting the significant prognostic factors in this cohort.

Our results come from one population of TIA patients who were referred to a neurologist with an interest in cerebrovascular disease and have been compared with one communitybased study in the same geographical area and other prospective hospital-referred series. Although care is needed in extrapolating the results to other populations of TIA patients, this sample of patients is probably quite similar, in terms of age, prevalence of vascular 
diseases and risk factors and prognosis, to other hospital-referred populations and can be used in planning diagnostic strategies, treatment, and treatment trials in such populations.

\begin{abstract}
Appendix
Definitions

Transient Ischaemic Attack (TIA): an acute loss of focal cerebral or monocular function with symptoms lasting less than 24 hours and which after adequate investigation was presumed to be due to embolic or thrombotic vascular disease. Neurological signs of no functional significance (for example, reflex asymmetry, extensor plantar response) may have been elicited subsequently. Non-focal neurological symptoms (such as, faintness) were acceptable only if accompanied by focal neurological symptoms. Symptoms occurring in isolation, such as vertigo, were not classified as TIAs. Patients in whom cranial CT demonstrated low attenuation areas appropriate to the clinical features were still classified as having a TIA on the basis of symptoms lasting less than 24 hours. ${ }^{41}$ Patients with lone bilateral blindness were excluded since, during their recruitment, we did not consider this symptom to be a TIA. ${ }^{45}$
\end{abstract}

Transient monocular blindness/amaurosis fugax ( $A F x)$ :

acute total or partial loss of vision in one eye with symptomatic recovery within 24 hours and after adequate investigation was presumed to be due to embolic or thrombotic vascular disease. Emboli may have been seen and there should not be any retinal or ocular pathology to account for the symptoms.

Definite carotid distribution TIA: amaurosis fugax or language dysfunction.

Definite vertebrobasilar distribution TIA: a combination of more than one of the following symptoms: diplopia, dysphagia, dysarthria, vertigo, bilateral simultaneous sensory and/or motor disturbance of the face and/or limbs, and bilateral or homonymous visual disturbance.

Hemiphenomena: unilateral weakness and/or sensory disturbance of the face and/or limbs plus or minus homonymous visual disturbance plus or minus dysarthria. Hemiphenomena associated with cerebral ischaemia are usually due to carotid territory ischaemia.

Minor stroke: patients with the clinical criteria of a stroke $e^{25-27}$ in whom symptoms lasted more than 24 hours and less than one week. Neurological signs of no functional significance (see above) were acceptable thereafter.

Major stroke: patients with the clinical criteria of a stroke ${ }^{25-27}$ in whom symptoms lasted more than one week or lead to an earlier death. The severity of the stroke was assessed approximately six months after onset by a modification of the Rankin scale; scores of 0 to 2 were considered "non-disabling" and scores of 3 to 5 were "disabling".

Non-fatal myocardial infarction: at least two of the following three criteria were required: 1) an appropriate clinical history of anterior chest pain, 2) development of pathological $Q$ waves on serial 12 lead ECGs, and 3) at least a $50 \%$ rise in serum cardiac enzymes.

Fatal myocardial infarction: as for non-fatal MI but necropsy evidence of definite infarction or a definite acute coronary artery thrombus in association with an appropriate clinical history.

Coronary Event (that is, ischaemic heart disease): included definite myocardial infarction, sudden death due to presumed or known ischaemic heart disease (on the basis of necropsy showing no other cause of death), and death due to cardiac failure secondary to ischeamic heart disease.

Vascular death: death due to stroke, myocardial infarction, sudden presumed cardiac death, cardiomyopathy, rheumatic heart disease, cardiac failure, ruptured aortic aneurysm, or pulmonary embolism.

Non-vascular death: death due to some disorder which is not related to vascular disease, for example, cancer, suicide, pneumonia.

Atheroma: a clinical diagnosis whereby structural change (such as hardening and thickening) in the arterial wall, causing luminal stenosis and thromboembolism, was the presumed cause of the target organ ischaemic syndrome in the absence of any evidence of other disorders such as inflammatory arterial disease, cardiogenic embolism, hypercoagulability and trauma.

Lipohyalinosis: a clinical diagnosis favoured by the clinical features of a "Lacunar" TIA. ${ }^{47}$

Cardiogenic embolism: a clinical diagnosis favoured by the occurrence of an isolated branch artery TIA or multiple TIAs in more than one arterial territory in either a relatively young patient (less than 50 years of age) with no other vascular diseases or risk factors, or a patient with a probable cardiac source of embolism demonstrated by clinical examination, electrocardiogram, chest radiograph or echocardiography.

Dr Hankey was supported by the Chest, Heart and Stroke Association and Mr Slattery by the Medical Research Council, 1 Marshall J. The natural history of transient ischaemic
cerebrovascular attacks. Q J Med 1964;33:309-24.
2 Baker RN, Ramseyer JC, Schwartz WS. Prognosis in
patients with transient cerebral ischaemic attacks.
Neurology 1968;18:1157-65.
3 Marshall J, Meadows S. The natural history of amaurosis
fugax. Brain 1968;91:419-34.
4 Acheson J, Hutchinson EC. The natural history of "focal
cerebral vascular disease". Q J Med 1971;40:15-23.
5 Goldner JC, Whisnant JP, Taylor WF. Long-term
prognosis of transient cerebral ischaemic attacks. Stroke
1971;2:160-7.
6 Ziegler DK, Hassanein RS. Prognosis in patients with
transient ischaemic attacks. Stroke 1973;4:666-73.
7 Ostfeld AM, Shekelle RB, Klawans HL. Transient
ischaemic attacks and risk of stroke in an elderly poor
population. Stroke 1973;4:980-6.
8 Whisnant JP, Matsumoto N, Elveback LR. Transient
cerebral ischaemic attacks in a community: Rochester,
Minnesota, 1955 through 1969. Mayo Clin Proc 1973;
48:194-8. 
9 Fields WS, Lemak NA. Joint study of extracranial arterial occlusion. IX. Transient ischaemic attacks in the carotid territory. JAMA 1976;235:2608-10.

10 Cartlidge NEF, Whisnant JP, Elveback LR. Carotid and vertebral-basilar transient cerebral ischaemic attacks: a community study, Rochester, Minnesota. Mayo Clin Proc 1977;52:117-20.

11 Heyden S, Heiss G, Heyman A, et al. Cardiovascular mortality in transient ischaemic attacks. Stroke 1980 11:252-5.

12 Simonsen N, Christiansen HD, Heltberg A, Marquardsen J, Pederson HE, Sorenson PS. Long-term prognosis afte transient ischaemic attacks. Acta Neurol Scand 1981; 63:156-68.

13 Muuronen $A$, Kaste $M$. Outcome of 314 patients with transient ischaemic attacks. Stroke 1982;13:24-31.

14 Heyman A, Wilkinson WE, Hurwitz BJ, et al. Risk of ischaemic heart disease in patients with TIA. Neurology 1984;34:626-30.

15 Hurwitz BJ, Heyman A, Wilkinson WE, Haynes CS, Utley $\mathrm{CM}$. Comparison of amaurosis fugax and transien cerebral ischaemia: a prospective clinical and arteriographic study. Ann Neurol 1985;18:698-704

16 Poole CJM, Ross Russell RW. Mortality and stroke after amaurosis fugax. J Neurol Neurosurg Psychiatry 1985; 48:902-5.

17 Howard G, Toole JF, Frye-Pierson J, Hinshelwood LC. Factors influencing the survival of 451 transient ischaemic attack patients. Stroke 1987;18:552-7.

18 Sorensen PS, Marquardsen J, Pedersen H, Heltberg A, Munck $O$. Long-term prognosis and quality of life after Munck O. Long-term prognosis and quality of life after 1989;79:204-13.

19 Dennis M, Bamford J, Sandercock P, Warlow C. The prognosis of transient ischaemic attacks in the community The Oxfordshire Community Stroke Project. Strok 1990;21:848-53.

20 Cutler SJ, Ederer F. Maximum utilisation of the life table method in analysing survival. J Chronic Dis 1958;8: 699-712.

21 Kaplan EL, Meier P. Nonparametric estimations from incomplete observations. American Statistical Association Journal 1958;53:457-81.

22 Howard G, Brockschmidt JK, Rose LA, et al. Changes in survival after transient ischaemic attacks: Observations comparing the 1970s and 1980s. Neurology 1989;39:982-5.

23 Gardner MJ, Altman DG. Statistics with confidence. Belfast, Universities Press, BMJ, 1989.

24 UK-TIA Study Group. United Kingdom transient ischaemic attack (UK-TIA) aspirin trial: interim results. ischaemic attack (UK-T

25 Bamford J, Sandercock P, Dennis M, et al. A prospective study of acute cerebrovascular disease in the community: the Oxfordshire Community Stroke Project-1981-1986. Methodology, demography and incident cases of first-ever
stroke. J Neurol Neurosurg Psychiatry 1988;51:1373-80.

26 Bamford J, Sandercock P, Dennis M, Burn J, Warlow C. A prospective study of acute cerebrovascular disease in the community: the Oxfordshire Community Stroke Project-1981-1986. Incidence, case fatality rates and overall outcome at one year of cerebral infarction, primary intracerebral haemorrhage and subarachnoid haemor rhage. J Neurol Neurosurg Psychiatry 1990;53:16-22.

27 Allen CMC. Clinical diagnosis of the acute stroke syndrome. $Q J$ Med 1983;208:515-23.

28 Sandercock $P$, Allen $C$, Corston $R$, Harrison M, Warlow C Clinical diagnosis of intracranial haemorrhage using
Guy's Hospital score. BMJ 1985;291:1675-8.

29 Bamford JM, Sandercock PAG, Warlow CP, Slattery JM Interobserver agreement for the assessment of handicap in stroke patients. Stroke 1989;20:828.

30 Bamford J, Sandercock P, Jones L, Warlow C. The natural history of lacunar infarction: the Oxfordshire Community Stroke Project. Stroke 1987;18:545-51.

31 Dennis MS, Bamford JM, Sandercock PAG, Warlow CP. Incidence of transient ischaemic attacks in Oxfordshire, England. Stroke 1989;20:333-9.

32 Dennis MS, Bamford JM, Sandercock PAG, Warlow CP. A comparison of risk factors and prognosis for transient ischaemic attacks and minor ischaemic strokes. The Oxfordshire Community Stroke Project. Stroke 1989; 20:1494-9.

33 Donnan GA, McNeil JJ, Adena MA, Doyle AE, O'Malley HM, Neill GC Smoking a risk factor for cerebral HM, Neill GC. Smoking a ris

34 Campbell A, Caird FI, Jackson TFM. Prevalence of abnormalities of electrocardiogram in old people. Br Heart $J$ 1974;36:1005-11.

35 Rose G, Baxter PJ, Reid DD, et al. Prevalence and prognosis of electrocardiographic findings in middle-aged men. $\mathrm{Br}$ Heart $J$ 1978;40:636-43.

36 Kannel WB, Abbott RD, Savage DD, et al. Epidemiological features of chronic atrial fibrillation: The Framingham Study. N Eng J Med 1982;306:1018-22.

37 Kraaijeveld CL, van Gijn J, Schouten HJA, Staal A. Interobserver agreement for the diagnosis of transient ischaemic attacks. Stroke 1984;15:723-5.

38 Koudstaal PJ, van Gijn J, Staal A, Duivenvoorden HJ, Gerritsma JGM, Kraaijeveld CL. Diagnosis of transient ischaemic attacks: improvement of interobserver ischaemic attacks: improvement of interobserver agreement by a ct

39 Bogousslavsky J, Regli F. Cerebral infarction with transient signs (CITS): do TIAs correspond to small deep infarcts in internal carotid artery occlusion. Stroke 1984;17:723-8.

40 Murros KE, Evans GW, Toole JF, Howard G, Rose LA Cerebral infarction in patients with transient ischaemic attacks. $J$ Neurol 1989;236:182-4

41 Dennis MS, Bamford JM, Sandercock PAG, Molyneux AJ, Warlow CP. Computerised tomography in patients with transient ischaemic attacks: When is a transient ischaemic attack not a transient ischaemic attack? J Neurol 1990; 237:257-61.

42 Wiebers DO, Whisnant JP, O'Fallon WM Reversible ischaemic neurologic deficit (RIND) in a community: Rochester, Minnesota, 1955-1974. Neurology 1982; 32:459-65.

43 Whisnant JP, Wiebers DO. Clinical epidemiology of transient cerebral ischaemic attacks (TIA) in the anterior and posterior cerebral circulation. In: Sundt $T M \mathrm{Jr}$, ed. Occlusive cerebrovascular disease, diagnosis and surgical management. Philadelphia: Saunders, 1987:60-65.

44 Antiplatelet Trialists' Collaboration. Secondary prevention of vascular disease by prolonged antiplatelet treatment. BMJ 1988;296:320-31.

45 Dennis MS, Bamford JM, Sandercock PAG, Warlow CP. Lone bilateral blindness: a transient ischaemic attack. Lancet 1989;i:185-8.

46 Hankey GJ, Warlow CP. Symptomatic carotid ischaemic events: safest and most cost-effective way of selecting patients for angiography, before carotid endarterectomy. patients for angiography,

47 Hankey GJ, Warlow CP. Lacunar transient ischaemic atuacts: a clincally useful concept? Lancet 1991;337:335-8. 